\section{Medo de queda recorrente e fatores associados em idosos de Florianópolis, Santa Catarina, Brasil}

\author{
Fear of recurrent falls and associated factors \\ among older adults from Florianópolis, \\ Santa Catarina State, Brazil \\ Miedo a caída recurrente y factores asociados en \\ ancianos de Florianópolis, Santa Catarina, Brasil
}

\begin{abstract}
Fear of falling is characterized by anxiety when walking or excessive worry about falling. This study aimed to investigate the factors associated with fear of recurrent falls among older adults in Florianópolis, Santa Catarina State, Brazil. A total of 266 older adults who had fallen in the previous year were studied based on a population-based cross-sectional survey. Statistical analysis used Poisson regression to assess the association between fear of recurrent falls and the covariates (socioeconomic variables, physical activity, diseases, cognitive impairment, socializing with friends, characteristics of falls, and selfrated health), respecting the hierarchy between the variables. Among the sample, 57.1\% feared recurrent falls. The adjusted analysis yielded $a$ significant association between the outcome and female gender ( $p=0.013)$, less socializing with friends $(p=0.015)$, diseases of the spinal column $(p=0.022)$, and limitation of daily activities after the fall ( $p=0.001)$. Thus, campaigns to prevent fear of new falls should particularly target women with limitations due to previous falls and low social interaction.
\end{abstract}

Aged; Accidental Falls; Fear
Danielle Ledur Antes 1

Ione Jayce Ceola Schneider 1

Tânia Rosane Bertoldo Benedetti 2 Eleonora d'Orsi 1

\section{Resumo}

O medo de cair caracteriza-se pela ansiedade ao caminhar ou preocupação excessiva em cair. Objetivou-se investigar os fatores associados ao medo de queda recorrente entre os idosos de Florianópolis, Santa Catarina, Brasil. Foram investigados 266 idosos de um estudo de base populacional que sofreram quedas no ano anterior. Para análise estatística utilizou-se a regressão de Poisson a fim de verificar a associação entre o desfecho "medo de queda recorrente" e as covariáveis (socioeconômicas, atividade física, doenças, déficit cognitivo, convívio com amigos, características da queda e percepção de saúde), respeitando-se a hierarquia entre as variáveis. Dentre os idosos indagados, $57,1 \%$ apresentaram medo de queda recorrente. Na análise ajustada obtevese associação significante entre o desfecho e ser do sexo feminino $(p=0,013)$, ter menor convívio com os amigos ( $p=0,015)$, doença da coluna $(p=$ 0,022) e limitações para atividades diárias após a queda $(p=0,001)$. Portanto, as campanhas de prevenção ao medo de nova queda devem visar prioritariamente às mulheres com limitação devido a quedas e com baixo convívio social.

Idoso; Acidentes por Queda; Medo 


\section{Introdução}

De acordo com relatório da Organização das Nações Unidas 1, em 2050 um quinto da população mundial terá 60 anos ou mais, e destes, $19 \%$ terão idade igual ou superior a 80 anos. Do ponto de vista da saúde, essa mudança na pirâmide etária está diretamente relacionada à transição epidemiológica, caracterizada pela alteração do perfil de morbimortalidade antes marcado pela alta prevalência de doenças transmissíveis, para o predomínio das doenças crônico-degenerativas e causadas por fatores externos, e suas complicações. O que muitas vezes significa maior demanda de utilização dos serviços de saúde - medicamentos, consultas médicas e internações hospitalares de longa duração, resultando, consequentemente, em maiores gastos 2 .

Dentre as enfermidades características desse novo perfil podem ser citadas as sequelas provocadas por quedas 2 . Acredita-se que em 2050, aproximadamente uma em cada três pessoas, com 65 anos ou mais, sofrerá uma ou mais quedas por ano, e cerca da metade destas resultará em lesões. Tanto as quedas como o medo de cair são síndromes comuns com resultados potencialmente graves em idosos 3 .

O medo de cair caracteriza-se pela ansiedade ao caminhar ou preocupação excessiva em cair. Fato que pode conduzir à menor confiança na capacidade de caminhar, depressão, sentimentos de desamparo, isolamento social, e alterações comportamentais que afetam a mobilidade funcional, promovem dependência física e até mesmo institucionalização precoce 4,5,6,7,8.

De acordo com Zijlstra et al. 7, a prevalência de medo de quedas varia de $20 \%$ a $85 \%$ entre idosos não institucionalizados. Estudos mencionam que o medo de cair está associado a piores condições de saúde, idade avançada, depressão, dificuldades nas atividades da vida diária (AVDs), lesões causadas por quedas, diminuição do convívio social, e estilo de vida sedentário 5,7,9,10. Embora haja uma crescente conscientização acerca do problema, pesquisas brasileiras são escassas. Assim, a realização deste estudo se justifica pela necessidade de evidenciar os fatores associados ao medo de cair em idosos brasileiros, mas especificamente em idosos de Florianópolis, município onde a população idosa representa mais de $10 \% 11$ e no qual não encontramos trabalhos sobre o tema desta investigação. Os resultados deste estudo poderão servir de base para o desenvolvimento de estratégias precoces de prevenção voltadas para o envelhecimento saudável da população alvo desta pesquisa. Portanto, o objetivo deste trabalho foi verificar os fatores associados ao medo de queda recorrente em idosos que relataram queda nos 12 meses anteriores à investigação.

\section{Métodos}

O estudo foi realizado valendo-se de um inquérito transversal, de base populacional e domiciliar, iniciado em 2009, intitulado EpiFloripa Idoso, desenvolvido na zona urbana do Município de Florianópolis, capital do Estado de Santa Catarina, Região Sul do Brasil. Florianópolis tinha uma população estimada, em 2009, de 408.161 habitantes, dos quais 44.460 idosos 11, ou seja, 10,8\% da população. É a capital brasileira com o melhor índice de desenvolvimento humano $(0,875) 12$.

No estudo sobre fatores associados ao medo de cair novamente foram selecionados somente os idosos que sofreram quedas no ano anterior à entrevista. O tamanho da amostra foi calculado a posteriori com o auxílio do programa OpenEpi version 2 (Dean AG, Sullivan KM, Soe MM. OpenEpi: Open Source Epidemiologic Statistics for Public Health, http://www.openepi.com). Os parâmetros utilizados foram: tamanho da população de 44.460 , prevalência do desfecho de $50 \%$, limite de confiança de 7,5\%, e efeito do delineamento de 2. Ao valor obtido foram acrescentados $10 \%$ para perdas, totalizando 375 entrevistados.

A amostra foi selecionada por conglomerados em dois estágios. No primeiro, todos os 420 setores censitários urbanos do município foram ordenados de maneira crescente, de acordo com a renda do chefe da família, sorteando-se sistematicamente 80 setores (oito em cada decil de renda).

As unidades do segundo estágio foram os domicílios. Uma etapa de atualização do número de domicílios em cada setor fez-se necessária, uma vez que o censo mais recente havia sido realizado em 2000. Para tanto, os supervisores do estudo percorreram os setores censitários sorteados e procederam à contagem de todos os domicílios habitados, obedecendo as normas do Instituto Brasileiro de Geografia e Estatística (IBGE). O número de domicílios nos setores variou de 61 a 725 .

A fim de diminuir o coeficiente de variação do número de domicílios por setor, realizou-se o agrupamento de setores com menos de 150 domicílios e a divisão dos que têm mais de 500, respeitando-se o decil de renda correspondente, originando 83 setores. O coeficiente de variação inicial era de $52,7 \%$ ( $n=80$ setores) e o final de $35,2 \%$ ( $\mathrm{n}=83$ setores).

Segundo o IBGE (Censo Demográfico 2000. http://www.ibge.gov.br/cidadesat/topwindow. htm?!, acessado em 12/Mai/2012), o número mé- 
dio de moradores por domicílio equivale a 3,1. Como as faixas etárias de interesse da pesquisa correspondem a aproximadamente $11 \%$ da população, obtém-se em média, por setor censitário, 102 pessoas na faixa etária de interesse ou um idoso a cada três domicílios. Portanto, deveriam ser visitados cerca de 60 domicílios por setor censitário, para se encontrar os 20 idosos. Esses domicílios foram sorteados de forma sistemática e todos os idosos residentes nos domicílios sorteados foram entrevistados.

Não foram incluídos os idosos institucionalizados (Instituições de Longa Permanência ou em hospitais). Considerou-se perda os idosos que não foram encontrados após quatro visitas, incluindo uma no fim de semana e outra à noite, e recusas quando os idosos se negaram a responder ao questionário.

A coleta de dados ocorreu entre setembro de 2009 e junho de 2010. Todas as variáveis do EpiFloripa Idoso foram coletadas por meio de um instrumento padronizado e pré-testado, utilizando-se personal digital assistants (PDA) As entrevistas foram realizadas por entrevistadoras com ensino médio completo. Todas participaram do processo de treinamento realizado pelos supervisores do inquérito, estudantes de pós-graduação, que também supervisionaram o trabalho de campo.

Com o intuito de verificar possíveis dificuldades de logística, inadequação do questionário e garantir a qualidade da coleta de dados, realizouse um estudo-piloto que incluiu 99 idosos, residentes em setores não amostrados para a pesquisa. Semanalmente verificou-se a consistência dos dados e o controle de qualidade, realizado em $10 \%$ das entrevistas, selecionadas aleatoriamente, por meio de um questionário reduzido aplicado por telefone.

Os idosos participantes do estudo EpiFloripa que responderam afirmativamente à questão: " $O$ (a) senhor(a) sofreu alguma queda no último ano?", foram selecionados para uma entrevista detalhada sobre a queda, na qual uma das questões fazia referência ao medo de cair novamente, que é definido por uma ansiedade ao caminhar devido à preocupação excessiva em cair 4 .

Para essa entrevista foi desenvolvido e testado um instrumento, com 21 questões, contendo perguntas sobre quantas vezes, onde, quando e como ocorreu a queda, se recebeu ajuda, quanto tempo permaneceu no chão, percepção sobre os fatores que motivaram a queda (tapetes, ambiente com pouca iluminação, piso escorregadio), uso de álcool e medicamentos antes da queda, consequências da queda (fratura, entorse, escoriação, perda de consciência), características do atendimento médico recebido após a queda, restrição ou dificuldade nas atividades da vida diária após a queda e medo de cair novamente. Esse questionário foi submetido ao processo de validação de conteúdo e clareza com um índice de validade de conteúdo de $97 \%$ e $100 \%$ de clareza 13 .

A investigação de quedas pode ser considerada um subestudo derivado do inquérito populacional EpiFloripa Idoso, que foi desenvolvido simultaneamente à entrega semanal das entrevistas do inquérito. Para responder à entrevista sobre quedas, os idosos foram contatados por meio de ligações telefônicas, e se isto não fosse possível eram realizadas visitas domiciliares. Essas entrevistas foram realizadas por três entrevistadoras, devidamente treinadas, seguindo o mesmo procedimento.

Para a investigação de quedas foram consideradas perdas os idosos não contatados após cinco ligações, realizadas em dias e horários distintos, seguidas de duas visitas domiciliares, ou quando se opuseram a participar. Caso o idoso não tivesse condições de responder à entrevista, ela era respondida por informante/cuidador. Foram excluídos da amostra os idosos que por motivo de demência, ou outro agravo, não conseguiam se comunicar para responder à questão referente ao medo de cair novamente por se tratar de uma questão subjetiva. Quedas em virtude de desmaios, acidente vascular cerebral (AVC), ataques epiléticos e afins não foram consideradas, sendo, portanto, excluídos três relatos. A investigação sobre a consequência da queda, quando mencionado mais de um tipo de lesão, optou-se pela mais grave, conforme percepção do entrevistado. Ao responder afirmativamente sobre maior dificuldade para realizar atividades após a queda, era possível fazê-lo com mais de uma alternativa, sendo que todas as opções foram consideradas para os resultados.

Foram extraídas do questionário do inquérito EpiFloripa Idoso as informações sobre quantidade de quedas nos 12 meses anteriores à entrevista (uma, de duas a quatro, cinco ou mais quedas); sexo; idade (60-69 anos, 70-79, 80 anos ou mais); cor da pele autorreferida (categorizada em brancos e negros/amarelos/indígenas agrupadas, devido ao baixo número de idosos); escolaridade em anos de estudos ( $\leq 4$ anos, $\geq 5$ anos); renda per capita em tercil (nível um: até $\mathrm{R} \$ 450,00$, nível dois: $\mathrm{R} \$ 450,01$ a R \$ 1.125,00, nível três: a partir de $\mathrm{R} \$ 1.125,01)$; atividade física no domínio de lazer verificada por meio da versão longa do Questionário Internacional de Atividades Físicas (IPAQ) 14,15 , categorizada em insuficientemente ativo (menos de 150 minutos semanais de atividades físicas no lazer) e suficientemente ativo 16; convívio com amigos categorizado em mais de uma 
vez por mês, uma vez por mês e raramente (uma vez por ano e nunca); déficit cognitivo investigado pelo Mini-Exame do Estado Mental (MEEM), categorizado em ausência de déficit cognitivo e provável déficit cognitivo, utilizando pontos de corte que levam em consideração o nível de escolaridade 17; doenças autorreferidas (doença de coluna, depressão, hipertensão, incontinência urinária, dor crônica) e autopercepção de saúde (muito boa/boa, regular, ruim/muito ruim).

Da investigação de quedas são oriundas as variáveis medo de cair novamente (sim/não), tempo no chão após a queda $(<1$ minuto, 2 a 14 minutos, > 15 minutos), perda da consciência após a queda (sim/não), atendimento no local da queda (sim/não), limitação para realizar atividades após a queda (sim/não) e fratura decorrente da queda (sim/não).

Para análise estatística utilizou-se o pacote estatístico do programa Stata SE, versão 9.0 (Stata Corp., College Station, Estados Unidos), considerando-se o efeito de desenho do estudo e os pesos amostrais. Foi empregada a estatística descritiva para os cálculos de prevalência, intervalos de 95\% de confiança (IC95\%), médias e desvios-padrão (DP). Para testar a associação entre o desfecho "medo de cair novamente (sim/ não)" definido como resposta positiva à pergunta "O(a) Sr.(a) tem medo de cair novamente?" e as covariáveis, realizou-se análise bruta e ajustada por meio da regressão de Poisson, sendo respeitada a hierarquia entre os possíveis fatores associados ao desfecho. As variáveis que apresentaram valor de $\mathrm{p} \leq 0,20$ na análise bruta e/ou ajustassem o modelo, foram selecionadas para entrar na análise ajustada. O seguinte modelo de análise foi empregado: nível 1 (distal): sexo, grupo etário e escolaridade; nível 2: atividade física no lazer e convívio com amigos; nível 3: déficit cognitivo, doença de coluna, hipertensão, depressão, incontinência urinária e dor crônica; nível 4: número de quedas nos doze meses anteriores ao estudo, tempo no chão após a queda e limitação nas atividades diárias após a queda; nível 5 (proximal): percepção de saúde. O efeito de cada exposição sobre o desfecho foi ajustado pelas demais variáveis do mesmo nível e dos níveis superiores.

O projeto foi submetido ao Comitê de Ética em Pesquisa com Seres Humanos da Universidade Federal de Santa Catarina (UFSC), sendo aprovado em 23 de dezembro de 2008 sob no $352 / 2008$. Todos os entrevistados assinaram o Termo de Consentimento Livre e Esclarecido para a realização da pesquisa.

\section{Resultados}

Entre os 1.705 entrevistados, 322 relataram ter sofrido queda no último ano (88 homens e 234 mulheres). Por causa das perdas e recusas, $82,6 \%$ desses foram indagados sobre medo de queda recorrente, sendo que $57,1 \%$ (IC95\%: 51,2; 63,1) responderam afirmativamente a esta pergunta. A prevalência de medo de quedas recorrentes entre o sexo feminino foi de $65,2 \%$; nos indivíduos com idades entre 60 e 69 anos este percentual chegou a $59,1 \%$; entre os idosos com quatro anos ou menos de estudos $65,8 \%$ referiram medo de cair novamente; dos entrevistados pertencentes ao segundo tercil de renda $64 \%$ mencionaram medo de novas quedas (Tabela 1).

Observou-se ainda, maior prevalência de medo de cair novamente entre idosos insuficientemente ativos no lazer, que relataram nunca ter contato com amigos, sem déficit cognitivo, com doença da coluna, artrite/reumatismo, diabetes, hipertensão, depressão, derrame/AVC, incontinência urinária, dor crônica e que relataram percepção de saúde ruim/muito ruim (Tabela 1).

Maiores prevalências de idosos com medo de quedas recorrentes foram encontradas entre aqueles que reportaram ter caído cinco ou mais vezes nos doze meses anteriores, ter permanecido no chão por no mínimo 15 minutos, não ter perdido a consciência em virtude da queda, nem necessitado de atendimento médico no local, ter sofrido consequência física em virtude da queda, possuir limitação para atividades da vida diária em função da queda e não ter sofrido fratura (Tabela 1).

$\mathrm{Na}$ análise bruta observou-se que o medo de quedas recorrentes foi significativamente associado com: ser do sexo feminino, escolaridade menor ou igual a quatro anos, raro convívio com os amigos, ter doença de coluna ou costas, depressão, incontinência urinária, dor crônica, ter sofrido cinco ou mais quedas, limitação das atividades após a queda e pior percepção de saúde (Tabela 1).

$\mathrm{Na}$ análise ajustada pode-se perceber que os idosos do sexo feminino apresentaram prevalência $79 \%$ maior de medo de quedas recorrentes, e quanto menor o convívio com os amigos maior a prevalência do desfecho. É possível verificar que idosos com doença de coluna têm prevalência $47 \%$ maior de medo de novas quedas. O relato de limitações nas atividades após a queda também se manteve associado, demonstrando que idosos que ficaram com limitações em virtude da queda tiveram prevalência $32 \%$ maior de relatarem medo de cair novamente (Tabela 2).

As variáveis, escolaridade, depressão, incontinência urinária, dor crônica e percepção 
Tabela 1

Análise bruta da associação entre medo de queda recorrente e as variáveis sociodemográficas, nível de atividade física no lazer, convívio com amigos, déficit cognitivo, morbidades referidas, características da queda e percepção de saúde. EpiFloripa Idoso 2009, Florianópolis, Santa Catarina, Brasil, 2011.

\begin{tabular}{|c|c|c|c|c|}
\hline Variáveis & n (\%) & $\begin{array}{c}\text { Medo de cair } \\
\text { n (\%) }\end{array}$ & $\begin{array}{l}\text { Análise bruta } \\
\text { RP (IC95\%) }\end{array}$ & Valor de $p$ \\
\hline Sexo & & & & 0,001 \\
\hline Masculino & $68(25,6)$ & $23(33,8)$ & 1,00 & \\
\hline Feminino & $198(74,4)$ & $129(65,2)$ & $2,17(1,36 ; 3,46)$ & \\
\hline Grupo etário (anos) & & & & 0,782 \\
\hline $60-69$ & $115(43,2)$ & $68(59,1)$ & 1,00 & \\
\hline $70-79$ & $111(41,7)$ & $65(58,6)$ & $1,09(0,83 ; 1,43)$ & \\
\hline$\geq 80$ & $40(15,0)$ & $19(47,5)$ & $0,88(0,56 ; 1,38)$ & \\
\hline Cor da pele & & & & 0,301 \\
\hline Brancos & $229(86,1)$ & $129(56,3)$ & 1,00 & \\
\hline Não brancos & $37(13,9)$ & $23(62,2)$ & $1,18(0,86 ; 1,62)$ & \\
\hline Escolaridade (anos) & & & & 0,026 \\
\hline$\geq 5$ & $147(55,3)$ & $74(50,3)$ & 1,00 & \\
\hline$\leq 4$ & $117(43,9)$ & $77(65,8)$ & $1,32(1,03 ; 1,66)$ & \\
\hline Renda per capita & & & & 0,490 \\
\hline Nível 1 & $106(39,8)$ & $63(59,4)$ & 1,00 & \\
\hline Nível 2 & $89(33,5)$ & $57(64,0)$ & $1,12(0,84 ; 1,48)$ & \\
\hline Nível 3 & $71(26,7)$ & $32(45,1)$ & $0,88(0,64 ; 1,21)$ & \\
\hline Atividade física no lazer & & & & 0,092 \\
\hline Suficientemente ativo & $55(20,7)$ & $22(40,0)$ & 1,00 & \\
\hline Insuficientemente ativo & $211(79,3)$ & $130(61,6)$ & $1,58(0,93 ; 2,68)$ & \\
\hline Convívio com amigos & & & & 0,028 \\
\hline Mais de uma vez por mês & $212(80,0)$ & $116(54,7)$ & 1,00 & \\
\hline Uma vez por mês & $30(11,3)$ & $18(60,0)$ & $1,20(0,92 ; 1,56)$ & \\
\hline Raramente & $23(8,7)$ & $17(73,9)$ & $1,34(1,00 ; 1,79)$ & \\
\hline Déficit cognitivo & & & & 0,142 \\
\hline Sim & $124(47,0)$ & $63(51,6)$ & 1,00 & \\
\hline Não & $140(53,0)$ & $87(62,1)$ & $1,20(0,94 ; 1,55)$ & \\
\hline Doença de coluna & & & & 0,001 \\
\hline Não & $90(33,8)$ & $40(44,4)$ & 1,00 & \\
\hline Sim & $176(66,2)$ & $112(63,6)$ & $1,82(1,29 ; 2,58)$ & \\
\hline Artrite/reumatismo & & & & 0,208 \\
\hline Não & $162(61,1)$ & $87(53,7)$ & 1,00 & \\
\hline Sim & $103(38,9)$ & $65(63,1)$ & $1,15(0,93 ; 1,42)$ & \\
\hline Diabetes & & & & 0,805 \\
\hline Não & $189(71,1)$ & $106(56,1)$ & 1,00 & \\
\hline Sim & $77(28,9)$ & $46(59,7)$ & $0,97(0,77 ; 1,22)$ & \\
\hline Hipertensão & & & & 0,055 \\
\hline Não & $93(35,0)$ & $44(47,3)$ & 1,00 & \\
\hline Sim & $173(65,0)$ & $108(62,4)$ & $1,40(0,99 ; 1,97)$ & \\
\hline Depressão & & & & 0,006 \\
\hline Não & $166(62,4)$ & $86(51,8)$ & 1,00 & \\
\hline Sim & $100(37,6)$ & $66(66,0)$ & $1,38(1,10 ; 1,74)$ & \\
\hline Derrame/AVC & & & & 0,660 \\
\hline Não & $236(88,7)$ & $134(56,8)$ & 1,00 & \\
\hline Sim & $30(11,3)$ & $18(60,0)$ & $0,90(0,57 ; 1,42)$ & \\
\hline
\end{tabular}

(continua) 


\begin{tabular}{|c|c|c|c|c|}
\hline Variáveis & $\mathrm{n}(\%)$ & $\begin{array}{l}\text { Medo de cair } \\
\mathrm{n}(\%)\end{array}$ & $\begin{array}{l}\text { Análise bruta } \\
\text { RP (IC95\%) }\end{array}$ & Valor de $p$ \\
\hline Incontinência Urinária & & & & 0,047 \\
\hline Não & $161(60,5)$ & $85(52,5)$ & 1,00 & \\
\hline Sim & $103(38,7)$ & $67(65,1)$ & $1,26(1,00 ; 1,59)$ & \\
\hline Dor crônica & & & & 0,002 \\
\hline Não & $127(47,7)$ & $55(43,3)$ & 1,00 & \\
\hline Sim & $139(52,3)$ & $97(69,8)$ & $1,59(1,19 ; 2,11)$ & \\
\hline Número de quedas & & & & $<0,001$ \\
\hline 1 & $157(59,0)$ & $81(51,6)$ & 1,00 & \\
\hline $2-4$ & $85(35,7)$ & $51(60,0)$ & $1,15(0,93 ; 1,43)$ & \\
\hline 5 ou mais & $24(5,3)$ & $20(83,3)$ & $1,76(1,43 ; 2,18)$ & \\
\hline Tempo no chão após a queda (minutos) & & & & 0,173 \\
\hline$\leq 1$ & $194(74,9)$ & $104(53,6)$ & 1,00 & \\
\hline $2-14$ & $45(17,4)$ & $29(64,4)$ & $1,24(0,92 ; 1,69)$ & \\
\hline$\geq 15$ & $20(7,7)$ & $13(65,0)$ & $1,16(0,82 ; 1,65)$ & \\
\hline Perda da consciência após a queda & & & & 0,403 \\
\hline Sim & $11(4,1)$ & $6(54,6)$ & 1,00 & \\
\hline Não & $255(95,9)$ & $146(57,3)$ & $1,26(0,73 ; 2,20)$ & \\
\hline Atendimento no local da queda & & & & 0,224 \\
\hline Sim & $10(3,8)$ & $5(50,0)$ & 1,00 & \\
\hline Não & $255(96,2)$ & $147(57,7)$ & $1,87(0,68 ; 5,14)$ & \\
\hline Limitação para realizar atividades após a queda & & & & $<0,001$ \\
\hline Não & $39(14,7)$ & $124(54,6)$ & 1,00 & \\
\hline Sim & $227(85,3)$ & $28(71,8)$ & $1,48(1,20 ; 1,82)$ & \\
\hline Fratura & & & & 0,424 \\
\hline Sim & $25(13,2)$ & $12(48,0)$ & 1,00 & \\
\hline Não & $165(86,4)$ & $102(61,8)$ & $1,22(0,74 ; 2,01)$ & \\
\hline Percepção de saúde & & & & 0,005 \\
\hline Boa & $91(34,2)$ & $41(45,1)$ & 1,00 & \\
\hline Regular & $127(47,7)$ & $77(60,6)$ & $1,55(1,08 ; 2,23)$ & \\
\hline Ruim & $46(17,3)$ & $33(71,7)$ & $1,66(1,15 ; 2,38)$ & \\
\hline
\end{tabular}

AVC: acidente vascular cerebral; IC95\%: intervalo de 95\% de confiança; RP: razão de prevalência.

de saúde não mantiveram no modelo multivariado a associação com o desfecho apresentada na análise bruta. Ao ser introduzida no modelo, a variável número de quedas apresentou associação significante com o desfecho, no entanto, ao serem inseridas as variáveis do nível seguinte, a quantidade de quedas saiu do modelo (Tabela 2).

\section{Discussão}

Nesta investigação, a maioria dos idosos que caíram relatou medo de quedas recorrentes $(57,1 \%)$, resultado que vai ao encontro de outros estudos 18,19,20. Ainda, de acordo com Lee et al. ${ }^{21}$, a maioria dos idosos desencadeia o medo de cair no- vamente após a experiência de queda com consequências mais graves, o que pode explicar a associação significante encontrada entre medo de cair e idosos que manifestaram ter sofrido, em virtude da queda, limitações nas suas atividades diárias. Tal associação também foi encontrada em estudo realizado na Austrália 21 sobre medo de cair novamente, no qual os idosos investigados passaram a limitar as atividades após a queda, encarando as atividades não essenciais (não relacionadas a auto-cuidado) como um risco de queda. Essas atividades, vistas como arriscadas mas essenciais para manter um estilo de vida independente, como se virar, sair da cama, ou caminhadas ao ar livre, passaram a ser realizadas em ritmo mais lento e com mais cuidado, devido ao medo de uma nova queda. 
Tabela 2

Análise ajustada da associação entre medo de quedas recorrentes e as variáveis sociodemográficas, nível de atividade física no lazer, convívio com amigos, déficit cognitivo, morbidades referidas, características da queda e percepção de saúde. EpiFloripa Idoso 2009. Florianópolis, Santa Catarina, Brasil, 2011.

\begin{tabular}{|c|c|c|c|c|c|c|c|c|c|c|}
\hline \multirow[t]{2}{*}{ Variáveis } & \multicolumn{2}{|l|}{$\begin{array}{l}\text { Bloco } 1 \\
(n=264)\end{array}$} & \multicolumn{2}{|c|}{$\begin{array}{c}\text { Bloco } 2 \\
(n=263)\end{array}$} & \multicolumn{2}{|l|}{$\begin{array}{l}\text { Bloco } 3 \\
(n=262)\end{array}$} & \multicolumn{2}{|l|}{$\begin{array}{c}\text { Bloco } 4 \\
(n=256)\end{array}$} & \multicolumn{2}{|c|}{$\begin{array}{l}\text { Bloco } 5 \\
(n=262)\end{array}$} \\
\hline & RP (IC95\%) & $\begin{array}{l}\text { Valor } \\
\text { de p }\end{array}$ & RP (IC95\%) & $\begin{array}{l}\text { Valor } \\
\text { de p }\end{array}$ & RP (IC95\%) & $\begin{array}{l}\text { Valor } \\
\text { de } p\end{array}$ & RP (IC95\%) & $\begin{array}{l}\text { Valor } \\
\text { de p }\end{array}$ & RP (IC95\%) & $\begin{array}{l}\text { Valor } \\
\text { de } p\end{array}$ \\
\hline Sexo & & 0,002 & & 0,002 & & 0,022 & & 0,011 & & 0,013 * \\
\hline Masculino & 1,00 & & 1,00 & & 1,00 & & 1,00 & & 1,00 & \\
\hline Feminino & $2,11(1,35 ; 3,36)$ & & $2,00(1,31 ; 3,04)$ & & $1,64(1,08 ; 2,51)$ & & $1,84(1,07 ; 1,42)$ & & $1,79(1,14 ; 2,82)$ & \\
\hline Grupo etário (anos) & & 0,713 & & & & & & & & \\
\hline $60-69$ & 1,00 & & & & & & & & & \\
\hline $70-79$ & $1,02(0,79 ; 1,31)$ & & & & & & & & & \\
\hline$\geq 80$ & $0,89(0,56 ; 1,41)$ & & & & & & & & & \\
\hline Escolaridade (anos) & & 0,069 & & 0,093 & & 0,335 & & & & \\
\hline$\geq 5$ & 1,00 & & 1,00 & & 1,00 & & & & & \\
\hline$\leq 4$ & $1,23(0,98 ; 1,54)$ & & $1,20(0,97 ; 1,49)$ & & $1,10(0,90 ; 1,35)$ & & & & & \\
\hline Atividade física no lazer & & & & 0,261 & & & & & & \\
\hline $\begin{array}{l}\text { Suficientemente } \\
\text { ativo }\end{array}$ & & & 1,00 & & & & & & & \\
\hline $\begin{array}{l}\text { Insuficientemente } \\
\text { ativo }\end{array}$ & & & $1,33(0,80 ; 2,21)$ & & & & & & & \\
\hline Convívio com amigos & & & & 0,009 & & 0,004 & & 0,004 & & 0,015 * \\
\hline $\begin{array}{l}\text { Mais de uma vez por } \\
\text { mês }\end{array}$ & & & 1,00 & & 1,00 & & 1,00 & & 1,00 & \\
\hline Uma vez por mês & & & $1,10(0,89 ; 1,36)$ & & $1,12(0,92 ; 1,37)$ & & $1,17(0,93 ; 1,49)$ & & $1,04(0,84 ; 1,30)$ & \\
\hline Raramente & & & $1,43(1,10 ; 1,85)$ & & $1,68(1,20 ; 2,33)$ & & $1,62(1,17 ; 2,24)$ & & $1,52(1,10 ; 2,11)$ & \\
\hline Déficit cognitivo & & & & & & 0,130 & & 0,063 & & 0,066 \\
\hline Sim & & & & & 1,00 & & 1,00 & & 1,00 & \\
\hline Não & & & & & $1,18(0,95 ; 1,47)$ & & $1,25(0,99 ; 1,57)$ & & $1,23(0,99 ; 1,52)$ & \\
\hline Doença de coluna & & & & & & 0,041 & & 0,015 & & 0,022 * \\
\hline Não & & & & & 1,00 & & 1,00 & & 1,00 & \\
\hline Sim & & & & & $1,46(1,02 ; 2,09)$ & & $1,53(1,09 ; 2,16)$ & & $1,47(1,06 ; 2,03)$ & \\
\hline Hipertensão & & & & & & 0,472 & & & & \\
\hline Não & & & & & 1,00 & & & & & \\
\hline Sim & & & & & $1,12(0,82 ; 1,54)$ & & & & & \\
\hline Depressão & & & & & & 0,352 & & & & \\
\hline Não & & & & & 1,00 & & & & & \\
\hline Sim & & & & & $1,11(0,89 ; 1,37)$ & & & & & \\
\hline Incontinência urinária & & & & & & 0,792 & & & & \\
\hline Não & & & & & 1,00 & & & & & \\
\hline Sim & & & & & $1,03(0,83 ; 1,28)$ & & & & & \\
\hline Dor crônica & & & & & & 0,241 & & & & \\
\hline Não & & & & & 1,00 & & & & & \\
\hline Sim & & & & & $1,18(0,89 ; 1,57)$ & & & & & \\
\hline Número de quedas & & & & & & & & 0,024 & & 0,135 \\
\hline 1 & & & & & & & 1,00 & & 1,00 & \\
\hline $2-4$ & & & & & & & $1,02(0,82 ; 1,27)$ & & $0,99(0,78 ; 1,26)$ & \\
\hline 5 ou mais & & & & & & & $1,41(1,18 ; 1,70)$ & & $1,32(1,08 ; 1,62)$ & \\
\hline
\end{tabular}

(continua) 


\begin{tabular}{|c|c|c|c|c|c|c|c|c|c|c|}
\hline \multirow[t]{2}{*}{ Variáveis } & \multicolumn{2}{|c|}{$\begin{array}{l}\text { Bloco } 1 \\
(n=264)\end{array}$} & \multicolumn{2}{|c|}{$\begin{array}{c}\text { Bloco } 2 \\
(n=263)\end{array}$} & \multicolumn{2}{|c|}{$\begin{array}{c}\text { Bloco } 3 \\
(n=262)\end{array}$} & \multicolumn{2}{|c|}{$\begin{array}{l}\text { Bloco } 4 \\
(n=256)\end{array}$} & \multicolumn{2}{|c|}{$\begin{array}{l}\text { Bloco } 5 \\
(n=262)\end{array}$} \\
\hline & RP (IC95\%) & $\begin{array}{l}\text { Valor } \\
\text { de } p\end{array}$ & RP (IC95\%) & $\begin{array}{l}\text { Valor } \\
\text { de } p\end{array}$ & RP (IC95\%) & $\begin{array}{l}\text { Valor } \\
\text { de } p\end{array}$ & RP (IC95\%) & $\begin{array}{l}\text { Valor } \\
\text { de } \mathrm{p}\end{array}$ & RP (IC95\%) & $\begin{array}{l}\text { Valor } \\
\text { de } \mathrm{p}\end{array}$ \\
\hline Tempo no chão após a & & & & & & & & 0,363 & & \\
\hline \multicolumn{11}{|l|}{ queda (minutos) } \\
\hline$\leq 1$ & & & & & & & 1,00 & & & \\
\hline $2-14$ & & & & & & & $1,30(1,06 ; 1,60)$ & & & \\
\hline$\geq 15$ & & & & & & & $0,95(0,65 ; 1,37)$ & & & \\
\hline Limitação para realizar & & & & & & & & 0,016 & & 0,001 * \\
\hline \multicolumn{11}{|l|}{ atividades após a } \\
\hline \multicolumn{11}{|l|}{ queda } \\
\hline Não & & & & & & & 1,00 & & 1,00 & \\
\hline Sim & & & & & & & $1,24(1,04 ; 1,50)$ & & $1,32(1,12 ; 1,57)$ & \\
\hline Percepção de saúde & & & & & & & & & & 0,075 \\
\hline Boa & & & & & & & & & 1,00 & \\
\hline Regular & & & & & & & & & $1,30(0,94 ; 1,81)$ & \\
\hline Ruim & & & & & & & & & $1,38(0,95 ; 1,99)$ & \\
\hline $\begin{array}{l}\text { Teste de Wald (valor } \\
\text { de p) }\end{array}$ & & 0,003 & & $\leq 0,001$ & & 0,001 & & $\leq 0,001$ & & $\leq 0,001$ \\
\hline
\end{tabular}

IC95\%: intervalo de 95\% de confiança; RP: razão de prevalência.

*Valores estatísticamente significantes $(\leq 0,05)$.

Os achados do presente trabalho demonstram associação significante entre medo de quedas recorrentes e sexo feminino, resultado corroborado por outras investigações 5,22,23,24. Segundo Fletchier \& Hirdes ${ }^{9}$, as mulheres tendem a superestimar os riscos de queda, enquanto os homens tendem a subestimar estes riscos, o que pode refletir respostas afirmativas em relação ao medo de cair.

Idosos com menor convívio com amigos também apresentaram maior prevalência de medo de quedas recorrentes; este resultado ilustra o que é citado por outras pesquisas. O medo de cair pode levar à restrição das atividades, possivelmente por causa da diminuição da autoconfiança sobre as habilidades, acarretando a redução do convívio social e isolamento, afetando de forma negativa a qualidade de vida dos idosos 5,8,25,26,27.

Estudos 28,29 apontam que doenças articulares são frequentes na população idosa e que contribuem para redução da capacidade física, influenciando negativamente no equilíbrio ou controle postural, o que talvez possa explicar a associação entre doença de coluna e medo de cair novamente. Porém, para melhor esclarecer tal relação são necessárias mais investigações acerca dessas variáveis, em virtude da falta de estudos que investigaram especificamente a relação entre doenças de coluna/costas e o medo de cair.
As principais limitações deste trabalho foram o fato de se tratar de um estudo transversal, o que impossibilita a ordem de causalidade entre as variáveis; o medo de cair ter sido investigado apenas por meio de uma questão, e somente entre os idosos que relataram quedas, assim como o número reduzido de sujeitos que participaram do subestudo, o que pode ter resultado na falta de associação entre algumas variáveis como idade avançada, depressão, sedentarismo e autopercepção de saúde; outra limitação que deve ser mencionada é o fato de terem sido excluídos da amostra as entrevistas referentes a idosos que por motivo de demência, ou outro agravo, não conseguiam se comunicar para responder à questão referente ao medo de cair novamente, no entanto, tendo em vista a subjetividade da questão esta não poderia ser respondida por familiares ou cuidadores. Em contrapartida, o porcentual de resposta, assim como o fato da amostra ser derivada de um estudo de base populacional da cidade de Florianópolis, aumentam a relevância do trabalho.

Os resultados encontrados mostram que mais da metade dos idosos investigados mencionou medo de cair novamente, sendo considerados fatores associados ao medo de quedas recorrentes: ser do sexo feminino, raro convívio com amigos, doença da coluna e relato de limitações para realizar atividades após a queda. 
Assim como apontam outras investigações $4,5,6,7,8$, os resultados encontrados mostram que o medo de cair deve ser encarado como uma preocupação de saúde pública, pois leva os idosos à maior propensão de declínio funcional e risco de quedas futuras, podendo conduzir à perda da independência e institucionalização precoce.

Portanto, os resultados sugerem que campanhas de prevenção ao medo de quedas recorrentes devem visar prioritariamente às mulheres que apresentam alguma limitação por causa de quedas e com baixo convívio social. Faz-se necessá- rio o desenvolvimento de programas de intervenção e políticas públicas, incluindo orientação aos profissionais de saúde, visando a evitar que o medo de cair torne-se responsável pela diminuição da qualidade de vida entre os idosos e seus familiares, os quais acabam se mobilizando para dar assistência ao idoso. O desenvolvimento de campanhas baseadas em esclarecimentos sobre os riscos que envolvem as quedas e o medo de quedas recorrentes, por meio de grupos de convívio com atividades físicas e discussões sobre o tema, pode ser uma importante ferramenta de prevenção aos serviços de saúde.

\section{Resumen}

El miedo a caerse se caracteriza por la ansiedad al caminar o preocupación excesiva en caerse. Se tuvo como objetivo investigar los factores asociados al miedo de caída recurrente entre los ancianos de Florianópolis, Santa Catarina, Brasil. Se investigaron a 266 ancianos de un estudio de base poblacional que sufrieron caídas durante el año anterior. Para el análisis estadístico se utilizó la regresión de Poisson, a fin de verificar la asociación entre el desenlace "miedo de caída recurrente" y las covariables (socioeconómicas, actividad física, en fermedades, déficit cognitivo, convivencia con amigos, características de la caída y percepción de la salud), respetándose la jerarquía entre las variables. Entre los ancianos entrevistados, un $57,1 \%$ presentaron miedo a caída recurrente. En el análisis ajustado se obtuvo una asociación significante entre el desenlace y ser del sexo femenino ( $p=0,013)$, tener menor convivencia con amigos ( $p=0,015)$, enfermedad de la columna ( $p=0,022)$ y limitaciones para actividades diarias tras la caída $(p=0,001)$. Por tanto, las campañas de prevención al miedo de una nueva caída deben tener como objetivo prioritariamente a las mujeres con limitacio nes, debido a las caídas, y con baja convivencia social.

Anciano; Accidentes por Caídas; Miedo

\section{Colaboradores}

D. L. Antes contribuiu para a concepção, planejamento, análise, interpretação dos dados, elaboração do rascu nho, revisão crítica do trabalho e aprovação da versão final. I. J. C. Schneider e E. d'Orsi contribuíram para a concepção, planejamento, análise e interpretação dos dados, revisão crítica do conteúdo e aprovação final do trabalho. T. R. B. Benedetti contribuiu para a concepção, planejamento e interpretação dos dados, revisão crítica do conteúdo e aprovação final do trabalho.

\section{Agradecimentos}

Agradecemos ao Instituto Brasileiro de Geografia e Estatística e à Secretaria Municipal de Saúde do Município de Florianópolis pelo auxílio para o desenvolvimento do estudo, e ao CNPq pelo apoio financeiro por meio de bolsa de pós-graduação. 


\section{Referências}

1. Programa das Nações Unidas para o Desenvolvimento. Atlas do desenvolvimento humano no Brasil, 2000. http://www.pnud.org.br/atlas/ (acessado em 13/Jun/2012).

2. Berenstein CK, Wajnman S. Efeitos da estrutura etária nos gastos com internação no Sistema Único de Saúde: uma análise de decomposição para duas áreas metropolitanas brasileiras. Cad Saúde Pública 2008; 24:2301-13.

3. Friedman SM, Munoz B, West SK, Rubin GS, Fried LP. Falls and fear of falling: which comes first? A longitudinal prediction model suggests strategies for primary and secondary prevention. J Am Geriatr Soc 2002; 50:1329-35.

4. Freiberger E, Vreede P. Falls recall - limitations of the most used inclusion criteria. Eur Rev Aging Phys Act 2011; 8:105-8.

5. Legters K. Fear of falling. Phys Ther 2002; 82: 264-72.

6. Pereira C, Vogelaere P, Baptista FT. Role of physical activity in the prevention of falls and their consequences in the elderly. Eur Rev Aging Phys Act 2008; 5:51-8.

7. Zijlstra GAR, van Haastregt JCM, van Eijk JTM, van Rossum E, Stalenhoef PA, Kempen GIJM. Prevalence and correlates of fear of falling, and associated avoidance of activity in the general population of community-living older people. Age Ageing 2007; 36:304-9.

8. Duque G, Kiel DP, Montero-Odasso M. Falls as a geriatric syndrome: how to prevent them? How to treat them? In: Duque G, Kiel DP, editors. Osteoporosis in older persons. London: Springer; 2009. p. 110-25.

9. Fletchier PC, Hirdes JC. Restriction in activity associated with fear of falling among communitybased seniors using home care services. Age Ageing 2004; 33:273-9.

10. Murphy SL, Dubin JA, Gill TM. The development of fear of falling among community-living older women: predisposing factors and subsequent fall events. J Gerontol A Biol Sci Med Sci 2003; 58:943-7.

11. Instituto Brasileiro de Geografia e Estatística. Estimativas populacionais para o TCU. Estimativas da população para 1o de julho de 2009. http://www. ibge.gov.br/home/estatistica/populacao/estima tiva2009/ (acessado em 12/Mai/2012).

12. Programa das Nações Unidas para o Desenvolvimento. Atlas do desenvolvimento humano no Brasil, 2003. http://www.pnud.org.br/atlas/ (acessado em 08/Mai/2012).
13. Antes DLA. Quedas e fatores associados em idosos de Florianópolis-SC: estudo EpiFloripa Idoso 2009 [Dissertação de Mestrado]. Florianópolis: Universidade Federal de Santa Catarina; 2011.

14. Benedetti TRB, Antunes PC, Rodriguez-Añez CR, Mazo GZ, Petroski EL. Reprodutibilidade e validade do Questionário Internacional de Atividade Fisica (IPAQ) em homens idosos. Rev Bras Med Esporte 2007 ; 13:11-6.

15. Benedetti TRB, Mazo GZ, Barros MVG. Aplicação do Questionário Internacional de Atividades Físicas para avaliação do nível de atividades físicas de mulheres idosas: validade concorrente e reprodutibilidade teste-reteste. Rev Bras Ciênc Mov 2004; 12:25-34.

16. Nelson ME, Rejeski JW, Blair SN, Duncan PW, Judge JO, King AC, et al. Physical activity and public health in older adults: recommendation from the American College of Sports Medicine and the American Heart Association. Circulation 2007; 116:1094-105.

17. Brucki SM, Nitrini R, Caramelli P, Bertolucci PH, Okamoto IH. Sugestões para o uso do mini-exame do estado mental no Brasil. Arq Neuropsiquiatr 2003; 61:777-81.

18. Iglesias CP, Manca A, Torgerson DJ. The health-related quality of life and cost implications of falls in elderly women. Osteoporos Int 2009; 20:869-78.

19. Pinheiro MM, Ciconelli RM, Martini LA, Ferraz MB. Risk factors for recurrent falls among Brazilian women and men: the Brazilian Osteoporosis Study (BRAZOS). Cad Saúde Pública 2010; 26:89-96.

20. Ribeiro AP, Souza ER, Atie S, Souza AC, Schilithz AO. A influência das quedas na qualidade de vida de idosos. Ciênc Saúde Coletiva 2008; 13:1265-73.

21. Lee F, Mackenzie L, James C. Perceptions of older people living in the community about their fear of falling. Disabil Rehabil 2008; 30:1803-11.

22. Boyd R, Stevens JA. Falls and fear of falling: burden, beliefs and behaviours. Age Ageing 2009; 38:423

23. Scheffer AC, Schuurmans MJ, van Dijk N, van Der Hooft T, de Rooij SE. Fear of falling: measurement strategy, prevalence, risk factors and consequences among older persons. Age Ageing 2008; 37:19.

24. Deshpande N, Metter EJ, Bandinelli S, Lauretani F, Windham BG, Ferrucci L. Psychological, physical and sensory correlates of fear of falling and consequent activity restriction in the elderly: The InCHIANTI Study. Am J Phys Med Rehabil 2008; 87:354. 
25. Suzuki M, Ohyama N, Yamada K, Kanamori M. The relationship between fear of falling, activities of daily living and quality of life among elderly individuals. Nurs Health Sci 2002; 4:155-61.

26. Austin N, Devine A, Dick I, Prince R, Bruce D. Fear of falling in older women: a longitudinal study of incidence, persistence, and predictors. J Am Geriatr Soc 2007; 55:1598-603.

27. World Health Organization. World Health Organization global report on falls prevention in olde age, 2007. http://www.who.int/ageing/publica tions/Falls_prevention7March.pdf (acessado em 15/Jun/2012).
28. Lima-Costa MF, Barreto SM, Giatti L. Condições de saúde, capacidade funcional, uso de serviços de saúde e gastos com medicamentos da população idosa brasileira: um estudo descritivo baseado na Pesquisa Nacional por Amostra de Domicílios. Cad Saúde Pública 2003; 19:735-43.

29. Fabrício SCC, Rodrigues RAP, Costa Junior ML. Causas e conseqüências de quedas de idosos atendidos em hospital público. Rev Saúde Pública 2004; 38:93-9.

Recebido em 08/Ago/2012

Versão final reapresentada em 14/Nov/2012

Aprovado em 28/Nov/2012 